\title{
FUCHS'S EPITHELIAL DYSTROPHY OF THE CORNEA*
}

\author{
BY \\ J. H. DOGGART \\ London
}

BEGINNERS in any branch of medicine are often accused of being too ready to diagnose rare diseases among patients afflicted with the common things. Bearing in mind, as we always should, that common diseases are often manifested non-typically, we must look with initial suspicion at any evidence pointing to a rarity. We know, for instance, that appendicitis can simulate all manner of recondite abdominal lesions, and that pneumonia emerging in bizarre forms may bewilder all but the most astute clinicians; and of course we have comparable pitfalls in ophthalmology. Nevertheless, I will not apologize for speaking about a rare condition. On the contrary, I want to emphasize the importance of Fuchs's corneal dystrophy. This disease is often missed, often enters into differential diagnosis, and it has been repeatedly confused with glaucoma. Therefore statistical tables concerning the relative frequency of eye diseases necessarily fail to convey the full significance of Fuchs's dystrophy in everyday clinical practice.

The main features of this malady are now well established. It affects both eyes, although one may be implicated earlier than the other, as obtains in so many bilateral diseases. Most of the victims are elderly, and women outnumber men in nearly all the recorded series. First the endothelium is attacked, then the epithelium, and finally the corneal substance. Failing vision is the cardinal symptom, and the causation remains obscure. Before we proceed to more detailed description, it will be convenient to recall the pioneer work upon this subject.

\section{BRIEF REVIEW OF THE LITERATURE}

Fuchs's original account of epithelial dystrophy (Fuchs, 1910) is a locus classicus in ophthalmic literature. There we find most lucidly marshalled all the essential points except those endothelial changes which were not recognizable until slit-lamp microscopy had been evolved. Fuchs describes the loss of corneal sensation, the development of epithelial vesicles spreading from the centre to the periphery of the cornea, and the subsequent invasion of the substantia propria. His paper is based upon thirteen elderly patients, nine of them women, set forth in detail. Although Fuchs did not possess that apparatus which enables us to scrutinize the living corneal endothelium, he was able to foreshadow that an endothelial defect might be responsible for this strange clinical entity. In his Bowman lecture delivered eight years earlier (Fuchs, 1902), he had already made brief mention of a

* Received for publication March 21, 1957. This communication is based on a paper delivered at the Spring 1957 Congress of the Gill Memorial Hospital, Roanoke, Virginia, U.S.A. 
strange condition simulating glaucoma in the corneae of elderly patients; and he speculated concerning the possibility of an endothelial change as the primary lesion. Those remarks, if they are read in conjunction with his 1910 paper, clearly show that Fuchs was already on the track of his now famous dystrophy, but he did not follow up this brilliant guess about the endothelium. The only fragment of cornea which he could secure for histological investigation was a disc consisting only of the superficial layers. Even a whole-thickness corneal section would probably have failed to display the endothelial changes after exposure to the chemical processes of fixation.

Kraupa (1920) was the first to publish a description of the endothelial changes which are now known to precede those of the epithelium in Fuchs's so-called epithelial dystrophy. It will be recalled that Gullstrand (1911) demonstrated his slit lamp in Heidelberg at the International Congress, just a year after the publication of Fuchs's classical paper. During the ensuing years, in the face of shortages and distractions created by the 1914-18 war, various improvements were wrought in Gullstrand's lamp. Kraupa made good use of these devices, and reached the correct conclusion that the endothelium is the first layer to be implicated in this disease. At the same time Vogt was scrutinizing the endothelial changes in Fuchs's dystrophy, though his observations were not published until the year after Kraupa's paper had appeared. Vogt (1921) gave, as might be expected, a beautifully vivid description, likening the altered endothelium to powdered bronze dusted upon the back of the cornea.

Vogt's powdered bronze phenomenon is essentially due to disintegration of the cells composing the endothelial mosaic, which are displayed by slit-lamp microscopy of the posterior corneal surface in the zone of specular reflection. In a minority of cases, endothelial disintegration is not the first sign produced by Fuchs's dystrophy. Instead, we may find large apparent holes in the endothelium of the axial region. Similar but smaller "holes" have been proved to consist of localized backward bulges of Descemet's membrane into the anterior chamber. Naturally the endothelium at the back of each bulge is thrown out of focus in relation to the rest of the endothelial sheet, and so we get the illusion of "holes". The same structural explanation holds good for the larger, axial "holes" occasionally found in early Fuchs's dystrophy. In this context it is interesting to recall the contribution of Graves (1924). Graves had assembled some bilateral cases of these multiple endothelial warts in elderly people, some of whom displayed epithelial vesicles; and he came to realize, when his paper was nearly completed, that he was dealing not with a new entity but with instances of Fuchs's dystrophy. Within the next year or two Friedenwald and Friedenwald (1925), Gifford (1925), and Sallmann (1926) all produced work to confirm that the endothelium is the first layer to be involved. Further striking testimony was supplied by Paufique (1951), who noted that attempts to relieve the victims by a superficial lamellar graft were foiled by transudation of aqueous through the abnormally permeable endothelium; but the question of surgical treatment will be considered later.

\section{Aetiology}

In the last quarter of a century I have been able to see at least a hundred instances of Fuchs's dystrophy, some of whom have been other people's 
patients sent for a second opinion. Of the 64 coming direetly under my care, 49 ( 73 per cent.) have been women, and fifteen ( 27 per cent.) men. In Fuchs's original but shorter series, the relative percentages were similar, viz. 69 for women, 31 for men. I know of no substantial collection of cases in which the females do not greatly outnumber the males, and it is now generally agreed that Fuchs's dystrophy is commoner in women than in men, although this aspect of the aetiology has not so far thrown any light upon the causation.

The next aetiological point to be considered is the age at onset. Among the first five patients I saw, the average age was 69 years. Some of them showed a late stage of the disease, and must already have been affected for years. Since then I have several times discovered early Fuchs's dystrophy during routine examination of patients not producing any symptoms. Therefore some reduction must be made from the average age of observed victims if we want to estimate the true average age at onset. Even after due allowance was made for this consideration, I was led to conclude that the disease was rare before the age of 60 years, and I never saw a single instance in a patient less than 50 years old until 12 years ago, when to my astonishment I found the whole fully-delineated picture in both eyes of a 36-year-old woman. Since then I have been able to see Fuchs's dystrophy in a further six patients under 40 years old. The youngest was a 34-year-old man free from symptoms, and 2 years later he had still experienced no difficulty, although the physical signs were certainly more pronounced. I often wonder whether the second world war has shattered a lot of our longestablished conceptions about aetiology. For example, most of us used to suppose that the older generation virtually monopolized basal-celled carcinoma of the eyelids, but since 1939 I have seen it in a number of patients less than 30 years old. We can still regard Fuchs's dystrophy mainly as a disease of old people, but it can no longer be ruled out as a possible explanation for visual failure in the lower age groups.

Having said that Fuchs's dystrophy is commoner in old than in young people, and that females are more susceptible than males, we arrive at the clouds of speculation if we continue to discuss aetiology. Treacher Collins (1907) described changes in the corneal epithelium associated with myxoedema and responding to the administration of thyroid extract, but this treatment fails to disperse Fuchs's dystrophy; and indeed these corneal lesions exhibit many points of difference from each other. Dystrophy of the corneal epithelium has also been found in cases of myotonia atrophica, but no particular systemic disease is typically linked with Fuchs's dystrophy; nor can Fuchs's corneal changes be dismissed as a manifestation of premature senility. Juler (1930) reported the disease in two sisters, but I know no other authentic example of familial incidence. No special climatic, occupational, or racial factors have been identified. 


\section{SYMPTOMS}

Fuchs's dystrophy, like most other bilateral diseases, may attack one eye sooner than the other, so that the earliest possible symptom is slight disturbance of vision in one eye. Sooner or later both eyes will become defective, but the march of disability may be exceedingly slow, and symptoms tend at first to be variable or even intermittent. Near objects look more blurred than distant ones, and many of the patients find that their sight improves as the day goes on.

When we recall the corresponding structural changes in the cornea we can perhaps account for this fluctuation. The endothelial changes, although they prepare the way for subsequent seepage of aqueous into the substance, do not of themselves obscure vision. Epithelial vesiculation, which becomes evident soon afterwards, is responsible for the blurring. Now it does not seem far-fetched to suppose that repeated blinks might temporarily clear the cornea by flattening the vesicles upon its epithelium. That supposition is borne out by clinical facts. Among several patients who had felt improvement in their sight as the day progressed, I was able to note that the signs as well as the symptoms were less obtrusive in the afternoon than in the morning. Evidently the eyelids as they move over the cornea with each blink produce an effect comparable with that of a light roller upon a piece of carefully clipped turf, so long as the vesicles remain small and delicate. As time goes on the epithelium coarsens, and changes develop in the corneal substance, so that vision remains bad all through the day.

Many of the patients suffering from Fuchs's dystrophy complain of seeing coloured rings around lights, a symptom which immediately raises the question of congestive glaucoma. It has been known for a long time that glaucomatous "haloes" are not the specific result of raised intra-ocular pressure. Epithelial vesicles, whatever may be their origin, can split white light into the colours of the Newtonian spectrum; and such vesicles can occur in a number of non-glaucomatous diseases, including Fuchs's dystrophy. Nevertheless the word "halo" continues to bias many ophthalmologists almost irrevocably towards a diagnosis of glaucoma. They see an elderly patient complaining of "haloes" and failing vision. Digital estimation of the intra-ocular pressure is notoriously uncertain. Even tonometry is far from precise. When once the mind has prematurely leapt to a diagnosis, rationalization can easily fill any gaps there may be in the evidence; and so it is not surprising that the literature contains numerous instances of people being wrongly subjected to drainage operations when they were in fact victims of Fuchs's dystrophy.

\section{Physical Signs}

Although the earliest signs of this disease are located in the endothelium, a large majority of the patients already show epithelial changes by the time 
that a diagnosis can be made. Early endothelial changes in Fuchs's dystrophy do not disturb vision, and remain undetected unless the patient happens to come under slit-lamp scrutiny. Not uncommonly, however, we see the disease limited to the endothelium in a second and less affected eye, when the other and earlier affected eye shows epithelial signs as well.

The commonest endothelial change is disintegration of the cells, so that, when we focus on the posterior surface of the cornea in the zone of specular reflection, there is no golden mosaic. The normal tessellation is replaced by those amorphous red-pepper granules which Vogt likened to powdered bronze. One source of fallacy must, however, be borne in mind. Slitlamp examination of elderly people nearly always reveals upon the hind surface of the cornea a few granules of iris pigment. Even in younger subjects we may discover similar particles aggregated into a Krukenberg's spindle; but this phenomenon should not be confused with endothelial dystrophy. Granules scattered by the iris pigment are never so abundant as to hide the whole of the endothelial sheet; and if we focus the posterior surface of the cornea in the zone of specular reflection away from the densest granular clumps, then we shall readily identify the mosaic pattern.

A minority of sufferers from Fuchs's dystrophy at first display large axial gaps in their endothelial tessellation. These apparent holes are, as we have already seen, due to localized backward bulges of Descemet's membrane together with the corresponding portion of endothelium. Although they do not directly impair the visual acuity, such interruptions in the endothelial mosaic are important in that they foreshadow more serious changes. Sooner or later the aforementioned endothelial disintegration will be evident, and by that time epithelial signs may have emerged.

Fine regular vesiculation in the axial region of the epithelium soon follows the endothelial changes in Fuchs's dystrophy. At this stage the patient may still be free from symptoms and in some instances the possibility of disease may be hinted by faint turbidity of the corneal axis examined with focal illumination and a uniocular loupe. The slit lamp will now clinch the diagnosis by displaying the characteristic endothelial lesions. It will be recalled that faint bedewing of the limbal epithelium is a physiological sign, but this entails no confusion with Fuchs's dystrophy, because the early epithelial changes in Fuchs's disease mainly implicate the axis, and fade away towards the periphery.

Alterations in the substantia propria are not long delayed when once the disease has invaded the endothelium and epithelium. Nearly all authorities agree that the main result of the endothelial disintegration is to render the hind layers of the cornea more permeable by the aqueous fluid. Swelling, striae, and irregular opacities, which typify Fuchs's dystrophy in the corneal substance, are attributable to aqueous percolating into and between the layers of the substantia propria.

Meanwhile, the anterior surface lesions are not stationary. The walls 
of adjacent epithelial vesicles tend to undergo absorption, thus forming bullae of variable size. When the larger blebs give way, corneal ulcer may be simulated, and sometimes the tags of loosened epithelium abortively proliferate, as in keratoconjunctivitis sicca (Sjögren's disease). These patients run the risk of being subjected to carbolization and other heroic remedies; and here let us insist that the victims of Fuchs's dystrophy respond badly to physical and chemical trauma.

Loss of corneal sensitivity is often mentioned as an important sign of Fuchs's dystrophy, but I suggest that we should write off this item of evidence for two reasons. First, its validity is questionable by reason of the many other forms of degenerative and inflammatory corneal trouble in which the surface sensitivity is impaired. Secondly, the diseased epithelium can readily become infected if we keep on jabbing it with pieces of cotton-wool merely to see whether sensation is registered. Another important source of danger is the tonometer, especially if this instrument be repeatedly planted upon the loose, vulnerable epithelium of patients suffering from Fuchs's dystrophy.

Advanced cases of this disease show dense opacity of the substantia propria, so that the endothelial lesion will be masked. Sooner or later, in patients who live long enough, the anterior layers of the cornea will undergo hyaline degeneration followed by calcareous change. Flakes of calcium compounds may be shed off into the conjunctival sac, and the corneal surface is irregular. Corneal ulcer can occur as a complication, but not as often as might be expected, considering the insensitive and friable state of the epithelium in Fuchs's dystrophy. Peripheral new vessels are a late and by no means invariable manifestation of the disease.

\section{Illustrative Case}

The case which I will now describe had been previously missed by two colleagues, but I am well aware that I must have been guilty of similar lapses in the past. People who come in search of a further opinion often put us on our mettle, because we can all feel a slight feline thrill at finding something which eluded the other doctor. This patient was a healthy-minded, busy, highly-educated woman, 67 years old, who had become increasingly aware, for the last year, that it was difficult for her to read The Times newspaper in the morning, although she could manage quite well later in the day. The ophthalmologist whom she first consulted assured her that there was nothing organically wrong, and he went on to advise that she had been overworking and would soon be restored by a holiday. Having found the situation unchanged when she came back from her holiday, she went to see a second ophthalmologist, but he deeply offended her by suggesting that a psychiatrist was the proper person to deal with her problem. She assured me that she had never felt any inclination to be a hypochondriac, and she described her matutinal reading handicap lucidly, without any neurotic overemphasis.

With small correcting-lenses each eye read $6 / 6$, and I found no abnormality in the fundi or media, but inspection of the corneae with the uniocular loupe and focal illuminator revealed faint but unmistakable turbidity of the axial surface. Then slit-lamp microscopy made the diagnosis certain. In both corneae the endothelium was amorphous, and fine regular vesicles covered the axial region of the epithelium. I told her that gentle eye massage through the closed lids for a few minutes every morning would probably be a help for some time to come; and in fact this simple palliative device did enable her to 
resume her morning study of the newspaper. Incidentally her joy at being released from the stigma of anxiety neurosis and the threat of psychiatry saved her from the disquiet which she would otherwise have felt about the revelation of eye disease.

DifFERENTIAL Diagnosis

We have already seen that advanced Fuchs's dystrophy can be confused with corneal ulcer and with Sjögren's disease, and that its early signs may easily be missed in the hurry of everyday clinical practice, but of course the most important diagnostic relationship is with glaucoma. Nowadays many of the glaucoma suspects inhabiting large cities will undergo minute investigation at special clinics. Provocative tests and other checks by trained observers have presumably lessened the chance of drainage operations being mistakenly done for eyes that exhibit Fuchs's dystrophy; but we must still be particularly vigilant when we are confronted with people in the higher age groups who complain about haloes and failing vision. Oedema of the corneal epithelium linked with these symptoms does not necessarily mean that there is or has recently been a rise in the intra-ocular pressure. Those who study the literature concerning Fuchs's dystrophy will find solid grounds for believing that the disease can readily be confused with glaucoma.

\section{TREATMENT}

Innumerable kinds of topical and systemic therapy have been tried for this disease. Dionine, holocaine, yellow oxide of mercury, hot applications, and various subconjunctival injections have all had their advocates. Sclerectomy, sclerotomy, iridectomy, lid suture, and grafting of mucous membrane after partial removal of the conjunctiva, are among the many procedures that have been tried; and one surgeon went so far as to apply tincture of iodine to the corneal surface after scraping away the epithelium. Systemically glycero-phosphates have been given, and many kinds of organo-therapy, including cortisone in recent years. The number and variety of these remedies coupled with the fact that most of them have fallen into desuetude supply ample comment.

Until recently there were only two ways in which one might help the people suffering from Fuchs's dystrophy. First of all, having established the diagnosis, one could ensure that they were not subjected to drastic topical measures, including drainage operations, likely to make them worse. Secondly, one could give simple advice about palliative measures, especially massage through the closed lids, and the discretional use of normal saline and parolein drops. Bearing in mind that the onset of Fuchs's dystrophy is usually late, we have the right to assume that many of the victims will die before being seriously handicapped by these slowly progressing corneal changes. Therefore palliative treatment is still important, but in the last few years the prospect of benefit from surgery has brightened.

We have already referred to the disappointing results of corneal grafting, whether whole-thickness or lamellar, in cases of Fuchs's dystrophy, even when those operations were done by grafting experts with a high record of 
success in other corneal conditions. Paufique (1953) became more and more convinced that permeability of the diseased endothelium was radically to blame in cases of Fuchs's dystrophy. Meanwhile he had achieved more success than at first he had dared to hope in certain cases of opacity involving the whole anterior corneal surface. He found that removal of the diseased layer was followed by total regeneration of the epithelium within a few days, and he conceived the hope that healthy endothelium might similarly re-form after removal of its diseased remnants. Animal experiments showed that Descemet's membrane and its endothelium could regenerate in just under 3 weeks. Paufique was well aware, however, that the membrane adheres firmly to the posterior layers of the substance. Therefore he was afraid that the upheaval entailed by scraping-off Descemet's membrane might preclude success. In fact it turned out that the attachment of Descemet's membrane is remarkably loosened in Fuchs's dystrophy. Therefore Paufique found it quite easy to scrape off the diseased posterior layers with a Meibomian curette passed through the temporal side of the limbus. Soon after this operation the substantia propria inevitably swells up, but that phase passes and the results have so far been encouraging. Paufique did not advocate this operation for slight, early instances of Fuchs's dystrophy, nor could we expect it to benefit those patients in whom all layers of the cornea display gross disease; but already there are good grounds for hope in properly selected cases of this hitherto hopeless condition.

\section{GeNERAL CONCLUSIONS}

Fuchs's dystrophy is a rare disease in a small organ, but there is far more to be said about it than has been written above. I could have given descriptions of a dozen illustrative cases instead of a single one. I might have dwelt at length upon the morbid histology, or perhaps discussed in greater detail the theories of origin, but many of you will be able to fill in gaps from your own experience or from your acquaintance with the literature. At any rate I hope I have been able to convince you, those who were not already convinced, that Fuchs's dystrophy is an important disease; and it is assuredly a humbling thought for us all that, in spite of research by generations of ophthalmologists in many lands, the origin of this condition is still an enigma.

\section{REFERENCES}

COLlins, E. TREACHER (1907). Trans. ophthal. Soc. U.K., 27, 47

FrIEDENWALD, H., and FrIEDENWALD, J. S. (1925). British Journal of Ophthalmology, 9, 14. FucHs, E. (1902). Trans. ophthal. Soc. U.K., 22, 15.

- (1910). v. Graefes Arch. Ophthal., 76, 478.

GifFORD, S. R. (1925). Arch. Ophthal. (N.Y.), 54, 217.

Graves, B. (1924). British Journal of Ophthalmology, 8, 502.

Gullstrand, A. (1911). Ber. ophthal. Ges. Heidelberg, p. 374.

JULER, F. (1930). Trans. ophthal. Soc. U.K., 50, 118.

KraUPA, E. (1920). Z. Augenheilk., 44, 247.

PAUfiQue, L. (1951). Bull. Soc. franc. Ophtal., 64, 270. (1953). Ibid., 66, 338.

SallmanN, L. (1926). Z. Augenheilk., 58, 348.

Vogt, A. (1921). v. Graefes Arch. Ophthal., 106, 63. 